\title{
Application of Automatic Variable Password Technique in Das's Remote System Authentication Scheme Using Smart Card
}

\author{
C. Koner, Member, IACSIT, C. T. Bhunia, Sr. Member, IEEE and U. Maulik, Sr. Member, IEEE
}

\begin{abstract}
Remote systems authentication schemes need more research and investigation due to increasing of hackers and attacks with the population of wired and wireless traffic. All of the popular remote user and system authentication schemes are fixed authentication and provides only entity authentication, not provides any data authentication. Recently Das proposed a flexible remote systems authentication scheme using smart card [8] that checks authenticity of user as well as remote system.

In this paper, we show that Das's scheme is not withstand the modification attack, reverse XOR attack and adversary system attack. We have proposed Modified Das's scheme which serves as entity authentication as well as data authentication. We have applied Automatic Variable Password technique (AVP) to make the password unbreakable by changing it session to session. Application of AVP made the Das's scheme a Time Variant Authentication scheme that checks the authenticity of remote user time to time. We show that how Modified Das's scheme defenses modification attack, reverse XOR attack and adversary system attack.
\end{abstract}

Index Terms-Automatic Variable Password, Remote user, Remote system, Smart card, Time Variant Entity and Data Authentication.

\section{INTRODUCTION}

Remote user authentication in sending information is a great research challenge. Remote user authentication is made of two types - Entity Authentication and Data Authentication. In Entity Authentication remote system checks the authenticity of remote user by the entity (e.g. password, smart card etc) of user before the transmission of user message. Authenticity is verified by the theory of public key cryptography. But Data Authentication remote system checks the authenticity of remote user by user message after receiving the message.

Chandan Koner is an Assistant Assistant Professor in the Department of Computer Science and Engineering, Bengal Institute of Technology and Management, Santiniketan, P.O. Doranda, West Bengal, Pin-731236, India., he is pursuing $\mathrm{PhD}$ course. He is member of IACSIT and IAENG. (Phone No.+91-9434535556, email: chandan_durgapur@yahoo.com)

Chandan Tilak Bhunia is an Director, Bengal Institute of Technology and Management, Santiniketan, P.O. Doranda, West Bengal, Pin-731236, India. He is a Senior Member of IEEE and FIE.

(Phone No.+91-9434033157, email: ctbhunia@vsnl.com)

Ujjwal Maulik is currently a Professor in the Department of Computer Science and Technology, Jadavpur University, Kolkata, India. He is a Senior Member of IEEE.

(Phone No.91-33-24131766, email: ujjwal_maulik@yahoo.com).
Many different Data Authentication [1-3] and Entity Authentication [4-8] are studied separately in literature.

Remote password authentication scheme using smart card was first proposed by Chang and Wu in 1993 [5]. After that, several new remote user password authentication schemes with smart card have been proposed. Remote user authentication using smart card, introduced by Hwang and $\mathrm{Li}$ in 2005 [6], is an application of ElGamal's cryptosystem [7]. After that, few public-key based authentication techniques have been invented and improved. But all of the techniques check only the authenticity of user but can not check the authenticity of system. In 2006, Das et al [8] developed a flexible remote user authentication scheme using smart card that authenticates user as well as remote system.

The entire remote user authentication schemes are fixed authentication (no time variant authentication) and provide only Entity authentication, not providing any Data authentication. This motivates us to develop a scheme that will serve as data authentication as well as entity authentication. The Automatic Variable Password (AVP) technique [9] can be applied by changing the password from session to session to make the password unbreakable. In this paper, we proposed Modified Das's scheme which is application of AVP technique that will check the authenticity of user time to time throughout the accessing of the remote server and it serves as a data authentication as well as entity authentication. We also show that Das's scheme is vulnerable to different attacks and how brilliantly Modified Das's scheme defenses those attacks and enhances the efficiency of the scheme.

\section{REVIEW Of DAS'S REMOte SyStem AUthENTICATION SCHEME}

Das's Remote System Authentication is a mutual authentication scheme, which authenticates the remote user as well as the remote system. The user chooses a password $\left(\mathrm{PW}_{\mathrm{i}}\right)$. The user has no private or public key but the remote system has a primary secret key (x) and a secret number (y). This scheme consists of three phases: registration phase, authentication phase and password change phase.

In the registration phase, the user $\mathrm{U}_{\mathrm{i}}$ submits $\mathrm{PW}_{\mathrm{i}}$ to the remote system (RS) for registration. The RS computes $N_{i}=h$

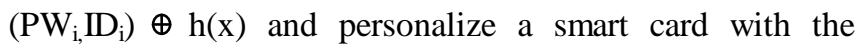
parameters $\mathrm{h}(),. \mathrm{N}_{\mathrm{i}} \mathrm{h}\left(\mathrm{PW}_{\mathrm{i}}, \mathrm{ID}_{\mathrm{i}}\right)$ and $\mathrm{y}$.

The authentication phase is divided into two parts, namely 
the User authentication and the RS authentication.

In user authentication phase, $\mathrm{U}_{\mathrm{i}}$ insert his smart card and submits $\mathrm{ID}_{\mathrm{i}}$ and $\mathrm{PW}_{\mathrm{i}}$. The smart card checks $\mathrm{PW}_{\mathrm{i}}$ and $\mathrm{ID}_{\mathrm{i}}$ with the stored ones in smart card If they are correct, the smart card computes $\mathrm{DID}_{\mathrm{i}}=\mathrm{h}\left(\mathrm{PW}_{\mathrm{i}} \mathrm{ID}_{\mathrm{i}}\right) \mathrm{h}(\mathrm{y} \mathrm{Tu})$, where Tu is timestamp of $\mathrm{U}_{\mathrm{i}}$ 's system and $\mathrm{C}_{\mathrm{i}}=\mathrm{h}\left(\mathrm{Ni} \quad \mathrm{y} \underset{\mathrm{Tu}}{\oplus}\right.$. $\oiint_{\oplus}$ egd $\left(\mathrm{DID}_{\mathrm{i}}, \mathrm{C}_{\mathrm{i}}, \mathrm{Tu}\right)$ as login request to the RS. RS receives the login request at time Ts and authenticates the $U_{i}$ by the following way. If the time interval between $\mathrm{Tu}$ and $\mathrm{Ts}$ is the expected valid time interval for the transmission delay, the $\mathrm{RS}$ computes $\mathrm{h}\left(\mathrm{PW}_{\mathrm{i}}, \mathrm{D}_{\mathrm{i}}\right)=$ $\mathrm{DID}_{\mathrm{i}} \oplus \mathrm{h}(\uplus \mathrm{Tu}) \oplus \mathrm{d} \bigoplus_{\mathrm{i}} *=\mathrm{h}\left(\mathrm{h}\left(\mathrm{PW}_{\mathrm{i}}, \mathrm{I}_{\mathrm{i}}\right) \oplus \mathrm{h}(\mathrm{x}) \oplus \mathrm{y} \oplus \mathrm{Tu}\right)$. If $\mathrm{C}_{\mathrm{i}} *=\mathrm{C}_{\mathrm{i}}$, the user is authentic.

In RS authentication phase, $\mathrm{RS}$ computes $\mathrm{X}_{\mathrm{i}}=\mathrm{h}\left(\mathrm{h}\left(\mathrm{PW}_{\mathrm{i}}, \mathrm{ID}_{\mathrm{i}}\right)\right.$ $\oplus \mathrm{h}(\mathrm{x}) \mathrm{Tu}$ Ts*) ${ }^{*} \mathrm{Th} *$ is timestamp of RS's system and sends $\left(\mathrm{X}_{\mathrm{i}}, \mathrm{Ts}^{*}\right)$ to the user. Let the user receives the response at time $\mathrm{Tu}^{*}$. If the time interval between $\mathrm{Tu}^{*}$ and $\mathrm{Ts} *$ is a valid time interval then computes $\mathrm{X}_{\mathrm{i}} *=\mathrm{h}(\mathrm{Ni} \mathrm{Tu} \mathrm{Ts} *)$. If $\mathrm{X}_{\mathrm{i}} *=\mathrm{X}_{\mathrm{i}}$, then the RS is authentic.

\section{CRyptanalysis Of Das'S Scheme}

We present a cryptanalysis of Das's remote system authentication scheme in this section. Das showed that, although his scheme is secured from replay, stolen verifier, impersonation, guessing and denial-of-service attack but his scheme is still vulnerable to modification attack, reverse XOR attack and adversary system attack. We demonstrate these attacks.

(i) Modification attack: As the remote system checks the authenticity of user before the transmission of message. Remote system does not verify the authentication of user by user message after receiving the message. So an adversary can change user message in the transmission of user message. Hence Das's scheme is suffer from modification attack.

(ii) Reverse XOR attack: During the registration phase, the secret key $\mathrm{x}$ has to be applied. Now based on the reversible property of XOR operation, if the primary secret key of remote system ( $\mathrm{x}$ ) is hacked and user password $\left(\mathrm{PW}_{\mathrm{i}}\right)$ is guessed, then nonce, $\mathrm{N}_{\mathrm{i}}$ can be easily obtained. Hence Das's scheme is vulnerable to the reverse XOR attack.

(iii ) Adversary system attack: Suppose the processors in remote system and card reader are very hasty and the transmission of data between user and server is happening in very speedily. In this type of communication, the timestamp of user $\mathrm{Tu}$ in user authentication phase and the timestamp of server Ts* in remote system authentication phase will be equal. During remote system authentication phase, remote system sends $\mathrm{X}_{\mathrm{i}}\left[=\mathrm{h}\left(\mathrm{N}_{\mathrm{i}}{ }^{\oplus} \mathrm{Tu}^{\oplus} \mathrm{Ts} *\right)\right.$ or $\mathrm{h}\left(\mathrm{h}\left(\mathrm{PW}_{\left.\mathrm{i}, \mathrm{ID}_{\mathrm{i}}\right)}\right.\right.$ $\oplus \mathrm{h}(\mathrm{x}) \stackrel{\oplus}{\oplus} \mathrm{Ts} *)]$ to the user over a public channel. As $\mathrm{Tu}$ and Ts*are same so $\mathrm{X}_{\mathrm{i}}=\mathrm{h}\left(\mathrm{N}_{\mathrm{i}}\right)$. Again suppose an adversary has stolen the user smart card for one time and just extract the $\mathrm{N}_{\mathrm{i}}$ and mode of hash function is known to him so he can easily compute $\mathrm{X}_{\mathrm{i}}$ which is $\mathrm{h}(\mathrm{x})$. Now if the adversary is also a user and accessing another server then he can send $\mathrm{X}_{\mathrm{i}}$ to the user by that illicit sever over the public channel before the original server sent. Then user can easily certify that server as an authentic server and communicates with that illicit server. The adversary, thus, can trick the user by connecting him with a wrong server. Das's scheme is therefore insecure from the adversary system attack.

\section{Modified Das'S Remote System Authentication SCHEME}

Modified Das's Remote System Authentication Scheme is mainly divided into two parts, namely, Entity Authentication Phase and Data Authentication Phase. The Entity Authentication Phase checks the authenticity of the remote user time to time by user password. The Data Authentication Phase checks the authenticity of the remote user by applying cryptography (Digital signature) on the user sending message. The Entity Authentication Phase is further subdivided into four parts, namely, User Enrollment Phase, User Login Phase, User Accessing Phase and Remote System Authentication Phase. Data Authentication Phase is also subdivided into three parts, namely, Key Generation Phase, Data Sending Phase and Data Receiving Phase.

\section{A. Entity Authentication Phase}

\section{1) User Enrollment Phase}

This phase is invoked whenever a user $U_{R}$ wants to register to the remote system $S_{R}$. The $U_{R}$ chooses a password $\mathrm{PW}_{1}$ and identifier ID and submits it to the $S_{R}$. After receiving enrollment request, the $S_{R}$ performs the following operations. DUE1: Computes, $\mathrm{N}=\mathrm{h}\left(\mathrm{PW}_{1} \oplus \mathrm{ID} \oplus \mathrm{x}\right)$, where $\mathrm{x}$ is a primary secret key of $S_{R}, h($.) is a one-way hash function and $\oplus$ is a bitwise concatenation operator.

DUE2: Personalizes a smart card $\mathrm{C}_{\mathrm{S}}$ with the parameters $\mathrm{h}(),. \mathrm{N}, \mathrm{PW}_{1}, \mathrm{ID}, \mathrm{E}, \mathrm{D}$ and $\mathrm{y}$, where $\mathrm{y}$ is the secondary secret number stored in each registered user's smart card, $\mathrm{E}$ is the encryption key and D is the decryption key generated by remote system by applying RSA algorithm.

DUE3: Sends the $C_{S}$ to the $U_{R}$ in a secure channel.

User Login Phase

When the $U_{R}$ wants to login to the $S_{R}$ then the following steps are executed. This part is executed only once when the $U_{R}$ wants to login to the $S_{R}$. $U_{R}$ inserts his $C_{S}$ and keys his identity ID and password $\mathrm{PW}_{1}$ '. The $\mathrm{C}_{\mathrm{S}}$ verifies the entered ID and $\mathrm{PW}_{1}{ }^{\prime}$ with the stored ones in $\mathrm{C}_{\mathrm{S}}$. If the ID and $\mathrm{PW}_{1}$ are correct, the $\mathrm{C}_{\mathrm{S}}$ executes the following steps,

DUL1: Computes, $\mathrm{D}=\mathrm{h}\left(\mathrm{PW}_{1} \oplus \mathrm{ID} \oplus \mathrm{x}\right) \oplus \mathrm{h}(\mathrm{y} \oplus \mathrm{Tu})$

DUL2: Computes, $\mathrm{C}=\mathrm{h}(\mathrm{N} \oplus \mathrm{Tu} \oplus \mathrm{y})$

Then send $(\mathrm{D}, \mathrm{C}, \mathrm{Tu})$ as login request to the $\mathrm{S}_{\mathrm{R}}$. Upon receiving the login request at time $T s$, the $S_{R}$ authenticates the $\mathrm{U}_{\mathrm{R}}$ the following steps,

DUL3: Computes, $\mathrm{h}\left(\mathrm{PW}_{1} \oplus \mathrm{ID} \oplus \mathrm{x}\right)=\mathrm{D} \oplus \mathrm{h}(\mathrm{y} \oplus \mathrm{Tu})$

DUL4: Computes, $\mathrm{C}^{*}=\mathrm{h}\left(\mathrm{h}\left(\mathrm{PW}_{1} \oplus \mathrm{ID} \oplus \mathrm{x}\right) \oplus \mathrm{y} \oplus \mathrm{Tu}\right)$

If $\mathrm{C}=\mathrm{C}^{*}$, the $\mathrm{S}_{\mathrm{R}}$ accepts the login request and gives permission to the $U_{R}$ to send the data.

2) User Accessing Phase

User accessing phase is executed to check authenticity of $U_{R}$ when $U_{R}$ is accessing the $S_{R}$. This phase is executed at a 
regular interval during the time of accessing the $S_{R}$ by $U_{R}$.

Let $T_{P}$ is timestamp of $S_{R}$ when the $U_{R}$ starts to access the $S_{R}$ and at a $\Delta \mathrm{T}$ regular interval the $\mathrm{S}_{\mathrm{R}}$ wants to verify the authenticity of $\mathrm{U}_{\mathrm{R}}$.

Now let $\mathrm{T}_{\mathrm{P}}+(\Delta \mathrm{T}+\ldots$

$$
\text { ..) }=\mathrm{T}_{\mathrm{P}}{ }^{\prime}
$$

Assume the $U_{R}$ 's message $M$ which is sent to the $S_{R}$, is a continuous bit stream. $\mathrm{C}_{\mathrm{S}}$ divides the $\mathrm{M}$ into different blocks of fixed size as the length of $\mathrm{PW}_{1}$ in Date sending phase of Data authentication phase. Let the message blocks are $\mathrm{M}_{1}, \mathrm{M}_{2}$, $\mathrm{M}_{3}, \ldots \ldots \ldots, \mathrm{M}_{\mathrm{n}}$

The $\mathrm{C}_{\mathrm{S}}$ generates modified blocks by the following way,

$\mathrm{PW}_{2}=\mathrm{PW}_{1} \oplus \mathrm{M}_{1}$,

$\mathrm{PW}_{3}=\mathrm{PW}_{2} \oplus \mathrm{M}_{2}$

So, $\mathrm{PW}_{\mathrm{n}}=\mathrm{PW}_{\mathrm{n}-1} \oplus \mathrm{M}_{\mathrm{n}-1}$

Thus the password at $\mathrm{i}^{\text {th }}$ position will be,

$\mathrm{PW}_{\mathrm{i}}=\mathrm{PW}_{\mathrm{i}-1} \oplus \mathrm{M}_{\mathrm{i}-1}$

$\mathrm{PW}_{\mathrm{i}}=\mathrm{PW}_{\mathrm{i}-2} \oplus \mathrm{M}_{\mathrm{i}-2} \oplus \mathrm{M}_{\mathrm{i}-1}$ and therefore

$\mathrm{PW}_{\mathrm{i}}=\mathrm{PW}_{1} \oplus \mathrm{M}_{1} \oplus \mathrm{M}_{2} \oplus \quad \ldots \ldots \ldots \ldots . . . \quad \mathrm{M}_{\mathrm{i}-2} \oplus \quad \mathrm{M}_{\mathrm{i}-1}$

The $\mathrm{C}_{\mathrm{S}}$ sends $\mathrm{PW}_{\mathrm{i}}$ blocks simultaneously as message blocks to $S_{R}$. The $C_{S}$ also uses $P_{\mathrm{i}}$ blocks one by one for every authentication checking execution after every $\Delta \mathrm{T}$ regular interval.

DUA1: The $S_{R}$ sends $\left\langle T_{P}{ }^{\prime}>\right.$ as an authentication query to the $\mathrm{C}_{\mathrm{S}}$ through a public channel after every $\Delta \mathrm{T}$ regular interval.

DUA2: After receiving the authentication query, the $\mathrm{C}_{\mathrm{S}}$ asks the $\mathrm{U}_{\mathrm{R}}$ to enter the ID and $\mathrm{PW}_{1}$.

DUA3: Then the $\mathrm{U}_{\mathrm{R}}$ enters his $\mathrm{ID}^{\prime}$ and $\mathrm{PW}^{\prime}{ }_{1}$.

DUA4: The $\mathrm{C}_{\mathrm{S}}$ validates the entered $\mathrm{ID}^{\prime}$ and $\mathrm{PW}^{\prime}{ }_{1}$ with the stored ones in $\mathrm{C}_{\mathrm{S}}$. If the ID and $\mathrm{PW}_{1}$ are correct then executes the following steps, otherwise terminates the accessing,

DUA4.1: Computes, $\mathrm{D}^{\prime}=\mathrm{h}\left(\mathrm{PW}_{\mathrm{i}} \oplus \mathrm{ID} \oplus_{\mathrm{X}}\right) \oplus \mathrm{h}\left(\mathrm{y} \oplus \mathrm{T}_{\mathrm{P}}{ }^{\prime}\right)$

DUA4.2: Computes, $\mathrm{C}^{\prime}=\mathrm{h}\left(\mathrm{N} \oplus \mathrm{y} \oplus \mathrm{T}_{\mathrm{P}}{ }^{\prime}\right)$

DUA4.3: Send ( $\left.D^{\prime}, C^{\prime}, T_{P}^{\prime}\right)$ as authentication request to the $S_{R}$ through a public channel.

DUA5: After receiving the authentication request, the $S_{R}$ authenticates the $\mathrm{U}_{\mathrm{R}}$ the following steps,

DUA5.1: Computes, $\mathrm{h}\left(\mathrm{PW}_{\mathrm{i}} \oplus \mathrm{ID} \oplus \mathrm{x}\right)=\mathrm{D}^{\prime} \oplus \mathrm{h}\left(\mathrm{y} \oplus \mathrm{T}_{\mathrm{P}}{ }^{\prime}\right)$

DUA5.2: Computes, $\mathrm{C}^{*} * \mathrm{~h}\left(\mathrm{~h}\left(\mathrm{PW}_{\mathrm{i}} \oplus \mathrm{ID} \oplus \mathrm{x}\right) \oplus \mathrm{y} \oplus \mathrm{T}_{\mathrm{P}}{ }^{\prime}\right)$

DUA5.3: Checks whether $\mathrm{C}^{\prime}=\mathrm{C}^{\prime *}$. If it holds, then gives permission to access again otherwise terminates the accessing.

3) Remote System Authentication Phase

Remote system authentication phase is executed to check the authenticity of $S_{R}$. The correctness of the $S_{R}$ is checked in this phase and executed when authenticity of $U_{R}$ is passed correctly.

DRA1: Computes, $\mathrm{X}=\mathrm{h}\left(\left(\mathrm{h}\left(\mathrm{PW}_{1} \oplus \mathrm{ID} \oplus \mathrm{x}\right) \oplus \mathrm{h}(\mathrm{Tu})\right) \oplus\right.$ $\mathrm{h}\left(\mathrm{Ts}^{*}\right)$ where $\mathrm{Ts} *$ is the timestamp of $\mathrm{S}_{\mathrm{R}}$.

DRA2: Send (X, Ts*) to the $\mathrm{U}_{\mathrm{R}}$ over a public channel.

The smart card computes $\mathrm{X}^{*}=\mathrm{h}((\mathrm{N} \oplus \mathrm{h}(\mathrm{Tu})) \oplus \mathrm{h}(\mathrm{Ts} *)$ and checks whether $\mathrm{X}=\mathrm{X}^{*}$ or not. If $\mathrm{X}=\mathrm{X}^{*}$, then $\mathrm{S}_{\mathrm{R}}$ is authentic and $U_{R}$ starts accessing the resources.

\section{B. Data Authentication Phase}

\section{1) Key Generation Phase}

Key Generation Phase is executed after receiving the enrollment request of $U_{R}$ by $S_{R}$ in parallel with user enrollment phase of Entity authentication phase. This phase is executed only once for one $U_{R}$. In this phase the $S_{R}$ performs the following steps,

DKG1: Generates Encryption Key (E) and Decryption Key (D) by RSA algorithm.

DKG2: Stores $\mathrm{E}$ and $\mathrm{D}$ into $\mathrm{C}_{\mathrm{S}}$ with other parameters $\mathrm{h}(),. \mathrm{N}$, $\mathrm{PW}_{1}, \mathrm{ID}$ and $\mathrm{y}$.

\section{2) Data Sending Phase}

Data sending phase is executed after the User login phase of Entity authentication phase. In this phase the $C_{S}$ performs the following steps,

DDS1: Divides the $M$ into different message blocks of fixed size as the length $\mathrm{PW}_{1}$. Let the message blocks are $\mathrm{M}_{1}, \mathrm{M}_{2}$, $\mathrm{M}_{3}, \ldots \ldots \ldots, \mathrm{M}_{\mathrm{n}}$.

DDS2: Sends the Decryption key D to the $S_{R}$ through a public channel

DDS3: Sends first message block $\mathrm{M}_{1}$ and the Digital Signature of $M_{1}$ (Calculated the Hash of $M_{1}$ then encrypts it by $E$ ) to the $\mathrm{S}_{\mathrm{R} \text {. }}$

DDS4: Generates $\mathrm{PW}_{2}, \mathrm{PW}_{3}, \ldots \ldots \ldots, \mathrm{PW}_{\mathrm{n}}$ blocks by the above theory.

DDS5: Sends $\mathrm{PW}_{2}, \mathrm{PW}_{3}, \ldots \ldots \ldots, \mathrm{PW}_{\mathrm{n}}$ blocks and Digital Signature of them to the $S_{R}$ through a public channel without waiting for authentication query from $S_{R}$. When $S_{R}$ sends authentication query after a $\Delta \mathrm{T}$ regular interval then stops the sending of $\mathrm{PW}_{\mathrm{i}}$. After authentication checking if $\mathrm{S}_{\mathrm{R}}$ gives permission to access then sends $\mathrm{PW}_{\mathrm{i}}$ blocks again simultaneously.

DDS6: If receives any request for sending any block again from $S_{R}$, then immediately sends it.

3) Data Receiving Phase

Data receiving phase is executed after receiving the first message block $M_{1}$ and the Digital Signature of $M_{1}$ from the $U_{R}$, the $S_{R}$ executes the following steps,

DDR1: Decrypts the Digital Signature of $M_{1}$ by $D$ and calculates the hash of $\mathrm{M}_{1}$. Then compares Digital Signature of $M_{1}$ and hash of $M_{1}$. If they are same, confirms that $M_{1}$ was sent from authentic $U_{R}$. If they are not same, rejects $M_{1}$.

DDR2: Then receives and decrypts the Digital Signature of $\mathrm{PW}_{2}, \mathrm{PW}_{3}, \ldots \ldots \ldots, \mathrm{PW}_{\mathrm{n}}$ by $\mathrm{D}$ and calculates the hash of them simultaneously. After that compares each decrypted block $\mathrm{PW}_{\mathrm{i}}$ with their hash. If any block is not same, rejects it.

DDR3: If any block is rejected then sends a request to $\mathrm{C}_{\mathrm{S}}$ for sending it again.

Now $\mathrm{S}_{\mathrm{R}}$ gets $\mathrm{M}$ by the following theory,

$\mathrm{M}_{2}=\mathrm{PW}_{2} \oplus \quad \mathrm{PW}_{3}$,

$\mathrm{M}_{3}=\mathrm{PW}_{3} \oplus \mathrm{PW}_{4}$,

So, $\mathrm{M}_{\mathrm{n}}=\mathrm{PW}_{\mathrm{n}} \oplus \mathrm{PW}_{\mathrm{n}+1}$

\section{SeCURity Analysis Of Modified Das'S SCHEME}

We analysis that how Modified Das's scheme is protected from the various security parameters. We discuss the defense of the scheme from the various attacks by which previous techniques are suffered.

Modification attack: In Modified Das's scheme the remote system checks the authenticity of user on user message after 
the transmission of message. If an adversary alters the authentic user message during the transmission of message, the remote system can easily identify it. Hence Modified Das's scheme is not suffer from Modification attack.

Reverse XOR attack: In this authentication scheme,

$\mathrm{N}[=\mathrm{h}(\mathrm{PW} \oplus \mathrm{ID} \oplus(\mathrm{x})]$ is computed in the registration phase. If $\mathrm{PW}$ is guessed and mode of hash function is leaked by an adversary, he never gets $\mathrm{N}$ because $\mathrm{N}$ is a function of four parameters PW, ID and x. Hence Modified Das's scheme is undoubtedly not vulnerable to the reverse XOR attack.

Adversary system attack: In remote system authentication phase, remote system sends $\mathrm{X}[=((\mathrm{h}(\mathrm{PW} \oplus \mathrm{ID} \oplus \mathrm{x}) \oplus \mathrm{h}(\mathrm{Tu}))$ $\oplus \mathrm{h}(\mathrm{Ts})]$ to the user over a public channel. For a very first system where Tu and Ts are same $\mathrm{X}$ will not be equal to the $\mathrm{h}$ (N). So if an adversary extracts the $\mathrm{N}$ by stoling the user smart card for one time and mode of hash function is known to him then he never gets X. So the user always authenticates a correct server. Hence Modified Das's scheme is firmly secured from the adversary system attack.

\section{EXPERIMENTAL RESULTS AND DISCUSSION}

Suppose $U_{R}$ submits the following password and identifier to the $S_{R}$,

User Password $\left(\mathrm{PW}_{1}\right)$ : User'sAuthentication

User Identifier (ID): IdentityofRemoteUser

Suppose $S_{R}$ generates Encryption and Decryption key by RSA algorithm in the following way,

Let, two large prime numbers $\mathrm{P}=13$ and $\mathrm{Q}=19$.

So, $\mathrm{N}=13 \times 19=247$

Encryption Key $(E)=31$ and Decryption Key $(D)=7$

Suppose $U_{R}$ sends a message of 1600 bits to the $S_{R}$,

User Message (M): "Authentication in sending information is a research challenge. Time Variant Authentication technique will check the authenticity of user for time to time throughout the accessing of the remote server."

$\mathrm{M}=$

$41757468656 \mathrm{e} 7469636174696 \mathrm{fbe} 20696 \mathrm{e} 207365$
$6 \mathrm{e} 64696 \mathrm{e} 6720696 \mathrm{e} 666 \mathrm{f} 726 \mathrm{~d} 6174696 \mathrm{f} 6 \mathrm{e} 206973$
$2061207265736561726368206368616 \mathrm{c} 6 \mathrm{c} 616 \mathrm{e} 67$
$65652054696 \mathrm{~d} 652056617269616 \mathrm{e} 742041757468$
$656 \mathrm{e} 7469636174696 \mathrm{f} 6 \mathrm{e} 20746563686 \mathrm{e} 69717565$
$2077696 \mathrm{c} 6 \mathrm{c} 20636865636 \mathrm{~b} 207468652061757468$
$656 \mathrm{e} 746963697479206 \mathrm{f} 66207573657220666 \mathrm{f} 72$
$2074696 \mathrm{~d} 6520746 \mathrm{f} 2074696 \mathrm{~d} 65207468726 \mathrm{f} 7567$
$686 \mathrm{f} 75742074686520616363657373696 \mathrm{e} 67206 \mathrm{f}$
$66207468652072656 \mathrm{~d} 6 \mathrm{f} 7465207365727665722 \mathrm{e}$

\section{A. Results of Entity Authentication}

The $\mathrm{C}_{\mathrm{S}}$ generates the following new passwords after receiving every authentication query from $S_{R}$,

$\mathrm{PW}_{2:}$ 1406111a421d351c170911071b0743081a491c0b

$\mathrm{PW}_{3}$ : 7a627874253d5c727166636a7a732a6774697578

$\mathrm{PW}_{4}$ : 5a035806404e391303050b4a191b4b0b18081b1f

$\mathrm{PW}_{5}:$ 3f2d785229235c335564792378753f2b597d6f77

$\mathrm{PW}_{6}$ : 5a430c3b4a42285a3a0a59571d165745300c1a12

$\mathrm{PW}_{7}$ : 7a34655726624b325f693277697e326551796e7a

$\mathrm{PW}_{8}$ : 1f5a113e450b3f4b7f0654571c0d5717711f0108
$\mathrm{PW}_{9}:$ 3f2e7853202b4b245f723d3a792d237f0370746f

$\mathrm{PW}_{10}: 57410 \mathrm{~d} 27005 f 23417 f 135 \mathrm{e} 591 \mathrm{c} 5 \mathrm{e} 50166 \mathrm{~d} 175400$

\section{B. Results of Data Authentication}

Digital Signature of Message of Das's Scheme is given below,

\section{$\mathrm{DF}=$ \\ c4a659b0cd0506e24d7c7c18534076cb4f33c7c1 e95f634bdcc11 ed63d47a29747753a9c8270d731 7 a 3 c 7883 c1 ca000a 544 cccc 9 af 4 fbd 631 bf 5007 a $6 \mathrm{~b} 7 \mathrm{a} 2 \mathrm{fc} 508054 \mathrm{adc} 5 \mathrm{aab} 03510142943407 \mathrm{a} 2 \mathrm{a} 62 \mathrm{a}$ be 39 a 369 d0 d 7ee 2 ff201 c6acca 9 a0939b 2 cdc 7 c806e7a6528209681e15e22fc75aa700 dad29 da8 $60 \mathrm{ca} 01 \mathrm{~d} 8 \mathrm{e} 6934601540 \mathrm{~b} 3 \mathrm{ac0b0e16a5739cf5d41}$ 92314 ed 220 d 9 f69 ae 082284 e 1202 ddd 984 e 8 ec 38 $5 \mathrm{a} 1 \mathrm{add} 213143767342 \mathrm{a} 693 \mathrm{~b} 7 \mathrm{c} 2 \mathrm{~d} 0 \mathrm{~d} 6 \mathrm{af8} 2 \mathrm{~b} 693$ ec665224121b7744ddb78ec067ee3 d30d354c07c}

Digital Signature of Message of Modified Das's Scheme is given below,

DT $=$

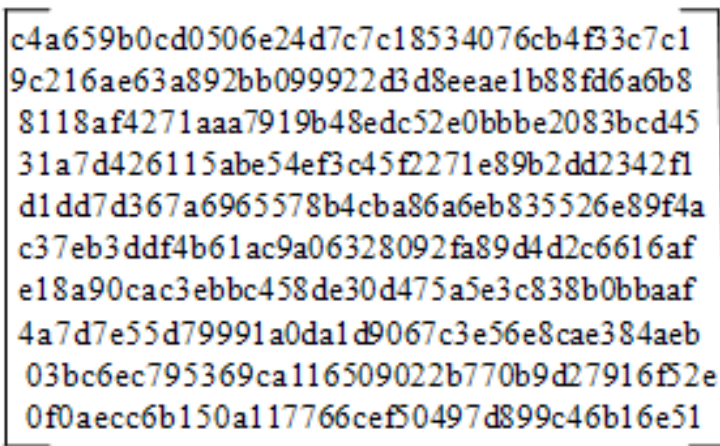

\section{EXPERIMENTAL ANALYSIS DAS'S SCHEME AND MODIFIED DAS'S SCHEME}

This section discusses the analysis of experiment results of the Das's scheme and Modified Das's scheme. We compare the techniques using three parameters: Distance, Redundant Character and Redundant Pair Character. Distance is defined as the summation of modulus of deviation between characters of plain text and cipher text. Redundant character measures the same character if they are in same position in plain text and cipher text. Redundant Pair character measures the pair of character if they are in same position in plain text and cipher text. 
A. Das's scheme

(i) Distance $=$ Deviation of $\mathrm{M}$ and $\mathrm{DF}=\sum$ Deviation of $\mathrm{m}_{\mathrm{ij}}$ from $\mathrm{df}_{\mathrm{ij}}=1937$.

The probability of authentication depends on the distance. Smaller distance increases the probability of authentication failure.

(ii) Redundant Character: If $\left(\mathrm{m}_{\mathrm{ij}}=\mathrm{df}_{\mathrm{ij}}\right)$ then the character is redundant. Here 28 characters are redundant out of 400 characters. So the probability of redundant character is $28 / 400$. The probability of redundant character increases the probability of authentication failure.

(iii ) Redundant Pair Character: If $\left(\mathrm{m}_{\mathrm{ij}} \mathrm{m}_{\mathrm{i}+1 \mathrm{j}+1}=\mathrm{df}_{\mathrm{ij}} \mathrm{df}_{\mathrm{i}+1 \mathrm{j}+1}\right)$ then the characters are redundant pair. Here 3 characters are redundant pair out of 200 characters. So the probability of redundant pair character is $3 / 200$. The probability of redundant pair character increases the probability of authentication failure.

\section{B. Modified Das's scheme}

(i) Distance $=$ Deviation of $\mathrm{M}$ and DT $=\sum$ Deviation of $\mathrm{m}_{\mathrm{ij}}$ from dt $_{\mathrm{ij}}=1979$.

The probability of authentication depends on the distance. Smaller distance increases the probability of authentication failure.

(ii) Redundant Character: If $\left(\mathrm{m}_{\mathrm{ij}}=\mathrm{dt}_{\mathrm{ij}}\right)$ then the character is redundant. Here 20 characters are redundant out of 400 characters. So the probability of redundant character is $1 / 20$. The probability of redundant character increases the probability of authentication failure.

(iii) Redundant Pair Character: If $\left(\mathrm{m}_{\mathrm{ij}} \mathrm{m}_{\mathrm{i}+1 \mathrm{j}+1}=\mathrm{dt}_{\mathrm{ij}} \mathrm{dt}_{\mathrm{i}+1 \mathrm{j}+1}\right)$ then the characters are redundant pair. Here 1 character is redundant pair out of 200 characters. So the probability of redundant pair character is $1 / 200$. The probability of redundant pair character increases the probability of authentication failure.

\section{Comparative Study By Result}

This section discusses comparison between the Das's scheme and Modified Das's scheme. We compare the techniques by the following parameters.

\section{A. Comparison by Distance}

The distance is lower in Das's scheme than Modified Das's scheme this means that the probability of authentication failure is more in Das's scheme. The distance is higher in Modified Das's scheme this means that the probability of authentication failure is lower Modified Das's scheme. Comparing by distance, we conclude that Modified Das's scheme is the more efficient authentication scheme.

\section{B. Comparison by Redundant Character}

The no of redundant character is lower in Modified Das's scheme than Das's scheme that means the probability of authentication failure is lower in Modified Das's scheme than Das's scheme. Comparing by redundant character, we conclude that Modified Das's scheme is the more efficient authentication scheme.

\section{Comparison by Redundant Pair Character}

The no of redundant character is lower in Modified Das's scheme than Das's scheme that means the probability of authentication failure is lower in Modified Das's scheme than Das's scheme. Comparing by redundant character, we conclude that Modified Das's scheme is the more efficient authentication scheme.

\section{CONCLUSION}

In this paper we have discussed the proposed Modified Das's remote system authentication scheme which checks the authenticity of remote user time to time and provides entity authentication as well as data authentication. This scheme is very fast operating since our proposed algorithm tested under C-programming. Therefore, this authentication method can be applied in real time basis for all sort remote network.

It has lot of advantages which are specifically listed below:

(i) This technique enjoys the advantages of Data Authentication as well as Entity Authentication.

(ii) An encrypted user message is sent to the remote system.

(iii) Insulated from modification attack, reverse XOR attack and adversary system attack.

(iv) User authenticity as well server authenticity is checked efficiently.

(v) Many users with same login identity can not able to $\log$ in.

(vi) Any user password database is not required in remote sever.

In future, we are exposing to find out more advanced realistic solution in the field of remote user and system authentication.

\section{REFERENCES}

[1] C. T. Bhunia, Information Technology Network and Internet, New Age International Publishers, India, $5^{\text {th }}$ Edition (Reprint), 2006.

[2] Charlie Kaufman, Radia Perlman and Mike Speciner, Network Security: Private Communication in Public World, Pearson Education, India, $2^{\text {nd }}$ Reprint, 2003.

[3] Atul Kahate, Cryptography and Network Security, Tata McGraw Hill, India, Sixth Reprint, 2006.

[4] L. Lamport, "Password authentication with insecure communication." Communication. ACM, Vol. 24, No. 11, pp. 770-772, 1981.

[5] C. C. Channg and T. C. Wu, "Rmote password authentication with smart cards", IEEE Proceeding-E, Vol. 138, no. 3,pp. 165-168, 1993.

[6] M. S. Hwang and L. H. Li, "A new remote user authentication scheme using smart cards", IEEE Transactions on Consumer Electronics, Vol. 46, No. 1, pp. 28-30, February 2000.

[7] T. ElGamal, "A public key based cryptosystem and a signature scheme based on discrete algorithms", IEEE Transactions on Information Theory, Vol. 31, No. 4, pp. 469-472, 1985

[8] M.L.Das, "Flexible and Secure Remote Systems Authentication Scheme Using Smart Cards". HIT Transaction on ECCN, Vol. 1, No.2, pp.78-82, April 2006.

[9] C.Koner, C.T.Bhunia, U.Maulik, "An Efficient and Reliable Time Variant Three-Entity and Data Authentication of Remote User Using Smart card" on ITNG09, IEEE Computer Society, USA pp487-491. 